\title{
Hierarchical multiobjective model predictive control applied to a dynamic pickup and delivery problem*
}

\author{
A. Núñez, B. De Schutter, D. Sáez, and C.E. Cortés
}

If you want to cite this report, please use the following reference instead:

\begin{abstract}
A. Núñez, B. De Schutter, D. Sáez, and C.E. Cortés, "Hierarchical multiobjective model predictive control applied to a dynamic pickup and delivery problem," Proceedings of the 13th International IEEE Conference on Intelligent Transportation Systems (ITSC 2010), Madeira Island, Portugal, pp. 1553-1558, Sept. 2010.
\end{abstract}

Delft Center for Systems and Control

Delft University of Technology

Mekelweg 2, 2628 CD Delft

The Netherlands

phone: +31-15-278.24.73 (secretary)

URL: https: //www.dcsc.tudelft.nl

*This report can also be downloaded via https://pub. deschutter.info/abs/10_047.html 


\title{
Hierarchical Multiobjective Model Predictive Control Applied to a Dynamic Pickup and Delivery Problem
}

\author{
Alfredo Núñez, Bart De Schutter, Doris Sáez, Cristián E. Cortés
}

\begin{abstract}
A hierarchical multiobjective model based predictive control approach is presented for solving a dynamic pickup and delivery problem. The hierarchical multilayer structure of the system is used to decompose the optimization problem into smaller but more tractable subproblems. In the bottom layer, the dispatcher (re)routes the vehicles when a new request appears, and minimizes user and operator costs. As those two components are usually aimed at opposite goals, the problem is formulated and solved through multiobjective model predictive control. The dispatcher participates in the dynamic routing decisions by expressing his/her preferences in a progressively interactive way. An illustrative experiment of the new approach through simulation of the process is presented to show the potential benefits in the operator cost and in the quality of service perceived by the users.
\end{abstract}

\section{INTRODUCTION}

The dynamic pickup and delivery problem (DPDP) considers a set of online requests of service for passengers traveling from an origin (pickup) to a destination (delivery) served by a fleet of vehicles initially located at several depots [1], [2], [3]. The final output of such a problem is a set of routes for the fleet, which dynamically change over time and are required in real-time. The DPDP designed to operate dial-aride systems (DARS) has been intensely studied in the last decades [4], [5], among which the ADART system in Corpus Christi Texas, which is a distributed system for dynamic routing already implemented in real-life [6].

A well-defined DPDP should be based on an objective function that includes prediction of future demands and traffic conditions in current routing decisions, [7]. In previous works we have proposed an analytical formulation for the DPDP as a model based predictive control (MPC) problem [8]. The resulting optimization problem was NP-hard, so, the use of evolutionary algorithms was considered. However, the global optimum solution was not reached due to the trade-off between computation time and accuracy in those algorithms. In this paper, we propose a new control structure for DPDP that does not only incorporate predictions, but also the inherent hierarchical multilayer and multiobjective structure of the DPDP.

Regarding hierarchical model based predictive control (HMPC), a very nice and comprehensive review can be found in [9]. In an HMPC structure the local actions of the

A. Núñez and B. De Schutter are with the Delft Center for Systems and Control, Delft University of Technology, Mekelweg 2, 2628 CD Delft, The Netherlands. $\{$ a.a. nunezvicencio, b. deschutter $\}$ etudelft.nl

D. Sáez and C.E. Cortés are with the Department of Electrical and Civil Engineering respectively, Universidad de Chile, Av. Tupper 2007, Santiago, Chile. $\{$ dsaez, ccortes\}@ing.uchile.cl controllers are coordinated by an algorithm operating at a higher level. The higher layers determine general characteristics of the system and generate control variables, usually by a static optimization procedure. In the lower layers, control variables are determined by means of a higher rate optimization procedure (MPC) and their effects are local and short-term. In this paper, we propose a hierarchical scheme with three layers for solving the DPDP.

In real implementations of DPDP the quality of service is very important. The authors in [10] conclude that most diala-ride studies are focused on the minimization of operational costs, and that it is necessary to develop more studies on user-policies. Therefore, we will consider quality of service for users while minimizing operational costs. We must notice that these two dimensions represent opposite objectives and we will need to solve conflicts between them. The users want to obtain good service, implying more direct trips, resulting in lower vehicle occupancy rates and consequently, higher operational costs. More efficient routing policies from the operators' standpoint will reflect higher occupation rates, longer routes, and consequently, longer waiting and travel time for users. Thus, to guide the decision-maker in this line, we propose the use of multiobjective model based predictive control. The dispatcher must express his/her preferences (criterion) in a progressive way (interactively), seeking the best-compromise solution from the dynamic Pareto set. Multiobjective optimization (MO) has been applied to vehicle routing problems [11], [12]. As far as we know, all the multiobjective applications in vehicle routing problems are evaluated in static scenarios, one of the aims of this paper being to contribute in the analysis of using MO in dynamic and stochastic environments.

The outline of the paper is as follows. In Section 2, the HMO-MPC approach is presented. In Section 3 the DPDP, including the model and the objective functions are discussed. In Section 4, the scheme based on MPC for solving the DPDP by [8] is reformulated under the new approach. In Section 5 simulation results are shown and analyzed. Finally conclusions and future work are highlighted.

\section{HIERARCHICAL MULTIOBJECTIVE MODEL PREDICTIVE CONTROL}

\section{A. Hierarchical Model Predictive Control}

In hierarchical multilayer systems, the system is divided into different functional layers, and the control structure consists of algorithms dealing with different components of the system, working at different temporal and spatial scales. This structure is useful to control plants characterized by 
significantly different dynamics and where the action of local controllers is coordinated by an algorithm operating at a higher level [9]. In some applications, at a higher level a simpler and more abstract model is considered to predict the long-term behavior of the system and to compute the optimal plant operating conditions based on an economic criterion. At the lower level, a more accurate model is used to compute the current control actions by looking at a shorter time horizon.

Consider for example a hierarchical model based predictive controller with a given number of layers. The variables of the higher layer are denoted with superscript 1 , in the next lower level the superscript is 2 and so on. The process modeled in layer $s$ is given by the following non-linear discrete-time system:

$$
x^{s}\left(k_{s}+1\right)=f_{s}\left(x^{s}\left(k_{s}\right), u^{s}\left(k_{s}\right), X^{s}\left(k_{s}\right)\right),
$$

where $x^{s}\left(k_{s}\right) \in \mathbb{R}^{n_{s}}$ is the state vector, $u^{s}\left(k_{s}\right) \in \mathbb{R}^{m_{s}}$ is the input vector, $X^{s}\left(k_{s}\right)$ are states and inputs from higher layers that affect the dynamics of layer $s$, and $k_{s} \in \mathbb{N}$ denotes the time step in layer $s$. In the layer $s$, the following MPC problem is solved:

\section{subject to}

$$
\min _{U^{s}} \lambda_{s} \cdot J_{s}\left(U^{s}, x_{k_{s}}^{s}\right)
$$

$$
\begin{aligned}
& x^{s}\left(k_{s}+\ell+1\right)=f_{s}\left(x^{s}\left(k_{s}+\ell\right), u^{s}\left(k_{s}+\ell\right), X^{s}\left(k_{s}+\ell\right)\right), \\
& \quad \ell=0,1, \ldots, N_{s}-1, \\
& X^{s}\left(k_{s}+\ell\right)=\left[x^{1}\left(k_{s}+\ell\right), \ldots, x^{s-1}\left(k_{s}+\ell\right),\right. \\
& \left.\quad u^{1}\left(k_{s}+\ell\right), \ldots, u^{s-1}\left(k_{s}+\ell\right)\right], \quad \ell=0,1, \ldots, N_{s}-1, \\
& x^{s}\left(k_{s}\right)=x_{k_{s}}^{s}, \\
& x^{s}\left(k_{s}+\ell\right) \in \mathbf{X}^{s}, \quad \ell=1,2, \ldots, N_{s}, \\
& u^{s}\left(k_{s}+\ell\right) \in \mathbf{U}^{s}, \quad \ell=0,1, \ldots, N_{s}-1,
\end{aligned}
$$

where $U^{s}=\left[u^{s}\left(k_{s}\right)^{\prime}, \ldots, u^{s}\left(k_{s}+N_{s}-1\right)^{\prime}\right]^{\prime}$ is the sequence of future control actions in layer $s, J_{s}\left(U^{s}, x_{k_{s}}^{s}\right)=$ $\left[J_{1}^{s}\left(U^{s}, x_{k_{s}}^{s}\right), \ldots, J_{l_{s}}^{s}\left(U^{s}, x_{k_{s}}^{s}\right)\right]^{\prime}$ are the $l_{s}$ objective functions to minimize, $\lambda_{s}=\left[\lambda_{s}^{1}, \ldots, \lambda_{s}^{l_{s}}\right]$ is the weighting factor vector, $N_{s}$ is the prediction horizon, $x^{s}\left(k_{s}+\ell\right)$ is the $\ell$-step-ahead predicted state from the initial state $x_{k_{s}}^{s}, X^{s}\left(k_{s}+\ell\right)$ is a vector with the predicted states and the outputs of higher layers that will affect the dynamics of layer $s$ at step $k_{s}+\ell$. We suppose $X^{s}\left(k_{s}+\ell\right)$ is known from the higher level MPC and is fixed for the optimization problem to solve in layer $s$. The state as well as the inputs are constrained to $\mathbf{X}^{s}$ and $\mathbf{U}^{s}$.

\section{B. Hierarchical Multiobjective Model Predictive Control}

Usually the objective functions in MPC are conflicting, i.e., a solution that optimizes one objective may not optimize others [13]. Multiobjective (MO) in MPC is a generalization of MPC, where instead of minimizing a single objective function, we consider more performance indices. In Hierarchical Multiobjective Model Predictive Control (HMO-MPC), if the layer $s$ modeled by (1) has conflicts, the following multiobjective problem is solved:

$$
\min _{U^{s}}\left\{J_{1}^{s}\left(U^{s}, x_{k_{s}}^{s}\right), J_{2}^{s}\left(U^{s}, x_{k_{s}}^{s}\right), \ldots, J_{l_{s}}^{s}\left(U^{s}, x_{k_{s}}^{s}\right)\right\}
$$

subject to the same constraints as in (2). The variables $U^{s}$ and $J_{l}^{s}\left(U^{s}, x_{k_{s}}^{s}\right), l=1, \ldots, l_{s}$, are the sequence of future control actions and the objective functions to minimize in layer $s$ respectively. The solution of HMO-MPC problem is a set of control action sequences called Pareto optimal set. Next we define Pareto optimality. Consider a feasible control sequence $U_{P}^{s}=\left[u_{P}^{s}\left(k_{s}\right)^{\prime}, \ldots, u_{P}^{s}\left(k_{s}+N_{s}-1\right)^{\prime}\right]^{\prime}$. The sequence $U_{P}^{s}$ is said to be Pareto optimal if and only if there does not exist another feasible control action sequence $U$ such that:

1) $J_{i}^{s}\left(U, x_{k}\right) \leq J_{i}^{s}\left(U_{P}^{s}, x_{k}\right)$, for $i=1, \ldots, l_{s}$.

2) $J_{j}^{s}\left(U, x_{k}\right)<J_{j}^{s}\left(U_{P}^{s}, x_{k}\right)$, for at least one $j \in\left\{1, \ldots, l_{s}\right\}$.

The Pareto optimal set contains all Pareto optimal solutions. The set of all objective function values corresponding to the solutions is known as the Pareto optimal front. From this set, just the first component $u^{s}\left(k_{s}\right)$ of one of those solutions has to be applied to the system, so at every instant, the controller (dispatcher in the context of a DPDP) has to use a criterion in order to find the control sequence that better suits the current objectives. In this paper, that decision is obtained after the Pareto set is determined. Then, it is not possible to choose a priori some weighting factor and to solve a single-objective optimization problem. The idea is to provide to the dispatcher a more transparent tool for the decisions.

\section{DYNAMIC PICKUP AND DELIVERY PROBLEM}

\section{A. Process description}

Dial-a-ride systems (DARS) are transit services which provide a shared-ride door-to-door service with flexible routes and schedules. The quality of service of a DARS is supposed to be in between of public transit buses and taxis. The typical specifications are the users pickup and delivery destinations and desired pickup or delivery times. We will assume that all the requests are known only after the dispatcher receives the associated call and that all the users want to be served as soon as possible. Thus, even we will not include explicitly hard windows, to provide a good service we propose a user-oriented objective function that deals with the problem of undesired assignments to clients, and keeps the service provided as regular (stable) as possible.

The service demand $\eta_{k}$ comprises the information of the request and is characterized by two positions, pickup $p_{k}$ and delivery $d_{k}$, the instant of the call $t_{k}$, a label $r_{k}$ that identifies the passenger who is calling and the number of passengers waiting there $\Omega_{k}$. Also we consider the expected minimum arrival time $t r_{k}$ which is the best possible time to serve the passenger, considering a straight journey from origin to destination (like a taxi service) and a waiting time obtained with the closest available vehicle (in terms of capacity) to pick up that passenger.

We assume a fixed and known fleet size $F$ over an urban area $A$. The dispatcher receives calls asking for service every instant $k$. Once a new request enters the system, the assignment of the vehicle and the insertion position of the new request into the previous sequence of that vehicle, are 
control actions decided by the dispatcher (controller), based on a dynamic objective function. Then, at any instant $k$, each vehicle $j$ is assigned to complete a sequence of tasks which includes several points of pickup and delivery. Only one of those vehicles will serve the last new request. The set of sequences $u(k)=S(k)=\left\{S_{1}(k), \ldots, S_{F}(k)\right\}$ correspond to the control variable. The sequence of stops assigned to vehicle $j$ at instant $k$ is given by $S_{j}(k)=\left[S_{j}^{0}(k), S_{j}^{1}(k), \ldots, S_{j}^{w_{j}(k)}(k)\right]$, where $S_{j}^{i}(k)$ is the information about the $i$-th stop and $w_{j}(k)$ is the number of planned stops of vehicle $j$ at instant $k$.

Two sources of stochasticity are considered: the first regarding the unknown future demand entering the system in real-time, and the second coming from the network traffic conditions, in its spatial and temporal dimension represented by a speed distribution $v(t, p)$ at instant $t$ in a position $p$. We will assume a conceptual network, where the trajectories are defined as the straight line that joins two consecutive stops. Besides, a speed distribution for the urban zone during a typical period represented by a speed model $\hat{v}(t, p)$ is supposed to be known, obtained from historical data.

\section{B. Process model}

The predictive model for vehicle $j$ is given by [8]:

$$
\begin{aligned}
& \hat{\chi}_{j}(k+1)=P_{j}^{i^{*}}(k)+\int_{T_{j}^{i^{*}}(k)}^{t_{k}+\tau} \frac{\hat{v}(t, p(t))\left(P_{j}^{i^{*}+1}(k)-P_{j}^{i^{*}}(k)\right)}{\left\|P_{j}^{i^{*}+1}(k)-P_{j}^{i^{*}}(k)\right\|} d t \\
& \hat{L}_{j}^{i}(k+1)=L_{j}^{0}(k)+\sum_{m=1}^{i}\left(2 z_{j}^{m}(k)-1\right) \Omega_{j}^{m}(k), \\
& \hat{T}_{j}^{i}(k+1)=t_{k}+\sum_{m=1}^{i} \kappa_{j}^{m}(k), \quad i=1, ., w_{j}(k) .
\end{aligned}
$$

where $\hat{\chi}_{j}(k)$ is the expected position of vehicle $j, \hat{T}_{j}^{i}(k)$ the expected departure time of vehicle $j$ from stop $i$, and $\hat{L}_{j}^{i}(k)$ the expected load of vehicle $j$ when leaving stop $i$. Moreover, $t_{k}$ is the continuous instant time when request $k$ happens, $\tau$ is the instant between $t_{k}$ and the occurrence of the future probable call, $i^{*}$ is the expected last stop visited by the vehicle before instant $t_{k}+\tau$, and $\kappa_{j}^{i}(k)$ is an estimation of the time interval between stop $i-1$ and stop $i$.

\section{Objective functions}

We design objective functions able to reflect the fact that some users can become particularly annoyed if their service is postponed (either pickup or delivery), by means of an incremental weight in the objective functions that penalizes differently very-long waiting or travel times.

The optimization variables are the current sequence $S(k)$ that incorporate the new request $\eta_{k}$, and the future sequences $S^{h}=\left\{S^{h}(k+1), \ldots, S^{h}(k+N)\right\}, h=1, \ldots, h_{\text {max }}$, that incorporate the prediction of future requests. The scenario $h$ consists of the sequential occurrence of $N-1$ estimated future request $\hat{\eta}_{k+1}^{h}, \hat{\eta}_{k+2}^{h}, \ldots, \hat{\eta}_{k+N-1}^{h}$, with a probability $p_{h}$. Thus $S_{k}^{k+N}=\left\{S(k), S^{1}, \ldots, S^{h_{\max }}\right\}$ comprises all the control actions to be calculated. The user cost $J_{1}(k)$ and the operator cost $J_{2}(k)$ are given by:

$$
\begin{aligned}
& J_{1}(k)=\sum_{j=1}^{F} \sum_{h=1}^{h_{\max }} \sum_{\ell=1}^{N} p_{h} \cdot\left(J_{j, h}^{U}(k+\ell)-J_{j, h}^{U}(k+\ell-1)\right) \\
& J_{j, h}^{U}(k+\ell)= \\
& \theta_{v} \sum_{i=1}^{w_{j}(k+\ell)} f_{r_{j}^{i}(k+\ell)}^{v}\left(1-z_{j}^{i}(k+\ell)\right)\left(\hat{T}_{j}^{i}(k+\ell)-t r_{r_{j}^{i}(k+\ell)}\right) \\
& +\theta_{e} \sum_{i=1}^{w_{j}(k+\ell)} f_{r_{j}^{i}(k+\ell)}^{e} z_{j}^{i}(k+\ell)\left(\hat{T}_{j}^{i}(k+\ell)-t_{r_{j}^{i}(k+\ell)}\right), \\
& J_{2}(k)=\sum_{j=1}^{F} \sum_{h=1}^{h_{\max }} \sum_{\ell=1}^{N} p_{h} \cdot\left(J_{j, h}^{O}(k+\ell)-J_{j, h}^{O}(k+\ell-1)\right) \\
& J_{j, h}^{O}(k+\ell)=c_{T}\left(\hat{T}_{j}^{w_{j}(k+\ell)}(k+\ell)-T_{j}^{0}(k+\ell)\right) \\
& \quad+c_{L} \sum_{i=1}^{w_{j}(k+\ell)} D_{j}^{i}(k+\ell),
\end{aligned}
$$

where $N$ is the prediction horizon, $h_{\max }$ is the number of predicted scenarios, $k+\ell$ is the instant at which the $\ell$-th request enters the system, measured from instant $k, p_{h}$ is the probability of occurrence of the $h$-th scenario, $J_{j, h}^{U}(\cdot)$ is the cost of the users in vehicle $j$, and $J_{j, h}^{O}(\cdot)$ is the operator cost of vehicle $j$ when the scenario $h$ occurs. The first component of $J_{j, h}^{U}(\cdot)$ is related to the re-routing time and the second component to the effective waiting time experienced by user $r_{j}^{i}(\cdot)$. Moreover, $f_{r_{j}^{i}(\cdot)}^{v}$ and $f_{r_{j}^{i}(\cdot)}^{e}$ are special weighting functions designed for the user $r_{j}^{i}(\cdot)$; both will start to grow linearly if the user is not experiencing a good total travel or a good waiting time respectively. Regarding $J_{j, h}^{O}(\cdot)$, it includes a first term that depends on the total operational time and another which depends on the total traveled distance. Thus, $D_{j}^{i}(\cdot)$ represents the distance between stops $i-1$ and $i$ in the sequence of vehicle $j$. Finally, $\theta_{v}, \theta_{e}, c_{T}$ and $c_{L}$ are weights defined by the dispatcher. In the next section, we propose to exploit the inherent hierarchical structure of this optimization problem, splitting the problem in smaller ones that work coordinated in different time scales.

\section{HMO-MPC FOR THE DPDP}

In Fig. 1 the proposed three layers scheme of HMO-MPC for DPDP is shown and next each layer is explained.

1) First layer, Dispatcher Management: The outputs of this layer are the parameters of the objective functions (4) and (5): value of waiting $\theta_{e}$ and travel $\theta_{v}$ times, value of each minute traveled by vehicles $c_{T}$, cost for kilometer traveled $c_{D}$, prediction horizon $N$, call-rate $\tau$. The demand patterns $h$ and their probabilities are determined here by using fuzzy clustering. Those parameters clearly change on time, each one with a different but slow rate. In this paper we are focusing on the operational process, so we will assume that the information provided by this layer is given and fixed.

2) Second layer, Vehicles Characterization: The second layer characterizes each vehicle according to its coverage area and occupancy by providing parameters of membership functions of a fuzzy inference system [14]. With this information, the controller determines the group of vehicles 


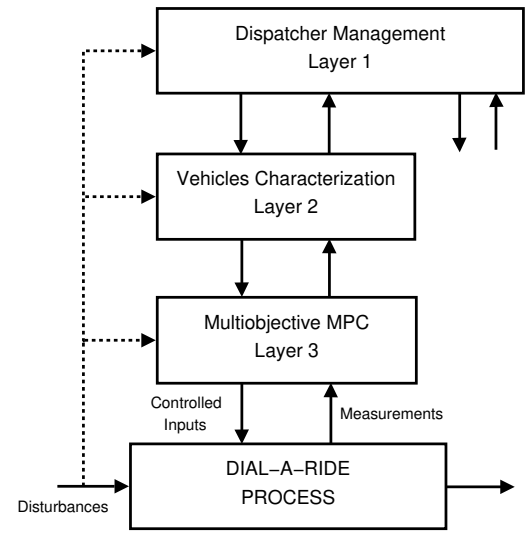

Fig. 1. HMO-MPC for the DPDP

with better chances to serve new requests. The information is updated every 20 minutes and will permit to reduce the computational effort when discarding vehicles too far away from new requests $\eta_{k}$ or when their number of tasks is too high. The output of this layer are the parameters of three membership functions (MFs) for each vehicle $j$, which represent the coverage in axis $x \mu_{x}^{j}(\cdot)$, in axis $y \mu_{y}^{j}(\cdot)$ and the number of tasks $\mu_{N}^{j}(\cdot)$. The following MFs are used:

$$
\begin{aligned}
& \mu_{x}^{j}\left(\eta_{k}^{x}\right)=\exp \left(-\frac{0.5\left(\eta_{k}^{x}-\bar{P}_{j}^{x}\right)^{2}}{\left(\sigma_{\sigma}^{x}\right)^{2}}\right), \quad \mu_{y}^{j}\left(\eta_{k}^{y}\right)=\exp \left(-\frac{0.5\left(\eta_{k}^{y}-\bar{P}_{j}^{y}\right)^{2}}{\left(\sigma_{j}^{y}\right)^{2}}\right) \\
& \mu_{N}^{j}\left(w_{j}(k)\right)=\frac{1}{1+e^{-\left(w_{j}(k)-c_{j}(t)\right)}}
\end{aligned}
$$

where $\eta_{k}^{x}$ and $\eta_{k}^{y}$ are the $x$ and $y$ coordinates of the pickup or delivery of the new request $\eta_{k}, \bar{P}_{j}^{x}$ and $\bar{P}_{j}^{y}$ are the mean values and $\sigma_{j}^{x}$ and $\sigma_{j}^{y}$ are the standard deviations of coordinates $x$ and $y$ of the task assigned to vehicle $j$ including the current position of the vehicle and the last stop visited. The variable $c_{j}(t)$ is the point of inflection of the sigmoidal membership function. The Gaussian MF for the coordinates captures the fact that some vehicles will serve requests in specific zones with a small coverage area, and others with a wider coverage. Regarding the sigmoidal (logit) shape of the MF for the number of tasks, the idea is to include the fact that when a vehicle is too saturated with future tasks, not only a bad service would be provided to the user, but also more computation time for solving the optimization problem would be required. We use $c_{j}(t)=10$ for the simulation results, so vehicles with up to 10 tasks are still reasonable.

3) Third layer, Multiobjective Optimization: The last layer consists of two components. The first one is a pre-processing algorithm where the optimization problem is reduced and conflicts between users and operator costs are detected. If there is a conflict, in the second component, we solve it by using MO-MPC.

The pre-processing algorithm is divided in three steps.

Step 1.1. Using a fuzzy inference system, the new request $\eta_{k}$ is evaluated in the MFs of each trip pattern. This fuzzy inference uses the parameters of MFs provided by the first layer. The predicted future scenarios with a high activation degree are chosen. These are denoted by $\bar{h}$.

Step 1.2. With another fuzzy inference system, the candidate vehicles $\bar{F}$ to serve the new and the probable requests $\bar{h}$ are determined. To show how this fuzzy inference works, consider for example the vehicle $j$ at instant $k$ as shown in Fig. 2. This fuzzy inference uses the parameters of the MFs provided by the second layer. A new call $\eta_{k}$ arrives, whose pickup coordinate is $(7,5)$ and whose delivery coordinate is $(7,6)$, as shown in Fig. 2(a). We check first whether $\eta_{k}$ is in the coverage area of vehicle $j$, by evaluating the MFs $\mu_{x}^{j}(\cdot)$ and $\mu_{y}^{j}(\cdot)$ shown in Fig. 2(b) and Fig. 2(c) respectively. For the pickup we get $\mu_{x}^{j}(7) \cdot \mu_{y}^{j}(5)=0.45$ and for the delivery $\mu_{x}^{j}(7) \cdot \mu_{y}^{j}(6)=0.53$. Then we check whether the number of stops by using the MF $\mu_{N}^{j}(\cdot)$ shown in Fig. 2(d). At instant $k, w_{j}(k)=10$, so $\mu_{N}^{j}(10)=0.5$, which means that the vehicle is still having a reasonable number of tasks. Finally the activation degrees of the rule for vehicle $j$ equals 0.12 . Whether vehicle $j$ is a good candidate or not, will depend on the conditions of the other vehicles. The first vehicle candidate is obtained by choosing the vehicle with the maximum activation degree, the second candidate with the second maximum, and so on (defuzzification).

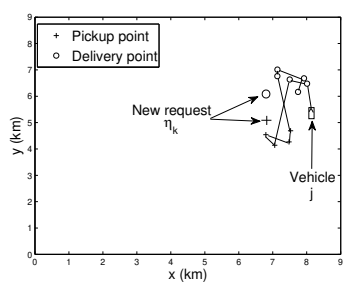

(a)

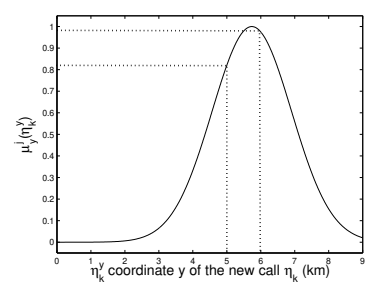

(c)

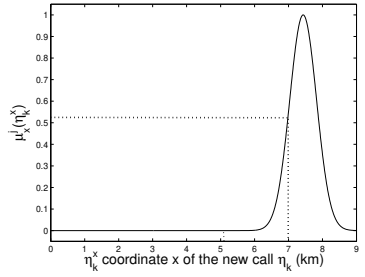

(b)

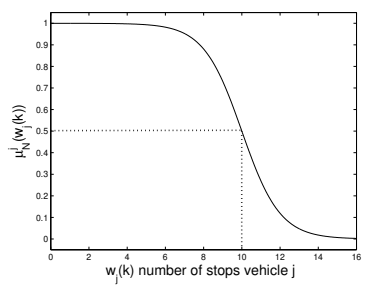

(d)
Fig. 2. Fuzzy characterization of vehicle $j$. (a) Vehicle $j$ sequence, Membership Functions for (b) x-axis, (c) y-axis and (d) number of stops

Step 1.3. Two MPC optimization problems are solved. To optimize just user cost and to optimize just operator cost:

$$
\begin{gathered}
\min _{S_{k}^{k+N}} J_{1}(k)=\sum_{j \in \bar{F}} \sum_{h \in \bar{h} \ell=1} \sum_{\ell}^{N} p_{h} \cdot \Delta J_{j, h}^{U}(k+\ell) \\
\text { s.t. } \quad \text { Model and constraints }
\end{gathered}
$$

$$
\begin{gathered}
\min _{S_{k}^{k+N}} J_{2}(k)=\sum_{j \in \bar{F}} \sum_{h \in \bar{h}} \sum_{\ell=1}^{N} p_{h} \cdot \Delta J_{j, h}^{O}(k+\ell) \\
\text { s.t. } \quad \text { Model and constraints }
\end{gathered}
$$

The objective functions minimized in (6) and (7), are like in (4) and (5) respectively, but considering just the set of 
vehicles $\bar{F}$ and the probable scenarios $\bar{h}$, which reduces the computational effort significantly. If the solution for both MPC problems (6) and (7) is the same or the trade-off between them is small, then the optimal solution which is closer to a pre-defined dispatcher criterion is used. If the trade-off is significant, then MO-MPC is required to find the optimal Pareto front, as the set of vehicles has conflicts and a better picture of the trade-off is necessary.

The MO-MPC algorithm is divided in four steps.

Step 2.1. The scenario $h$ consists of the sequential occurrence of $N$ requests $\eta_{k}, \hat{\eta}_{k+1}^{h}, \hat{\eta}_{k \pm 2}^{h}, \ldots, \hat{\eta}_{k+N-1}^{h}$. For each vehicle $j \in \bar{F}$, for each scenario $h \in h$, we will solve $2^{N}$ MO problems considering the cases where vehicle $j$ is the one that serves none, one, or a combination of more of those requests. For example, if $N=2$, for each vehicle we solve four MO problems considering the cases to serve none, to serve $\eta_{k}$, to serve $\hat{\eta}_{k+1}^{h}$, and to serve $\eta_{k}$ and $\hat{\eta}_{k+1}^{h}$. The MO problem in this step is the following:

$$
\min _{\left\{S_{j}(k), S_{j}^{h}(k+1), \ldots, S_{j}^{h}(k+N)\right\}}\left\{\sum_{\ell=1}^{N} \Delta J_{j, h}^{U}(k+\ell), \sum_{\ell=1}^{N} \Delta J_{j, h}^{O}(k+\ell)\right\}
$$

Capacity constraints and consistency are considered, so the Pareto set contains just feasible sequences. Note that some of those MO problems are easy to solve, but the more requests the vehicle serves, the more possible solutions we will have. In fact, considering the no-swapping constraint, the number of possible solutions when the $N$ requests are served by vehicle $j$ only is $0.5 \cdot \prod_{i=0}^{N-1}\left(w_{j}(k)+i\right)\left(w_{j}(k)+i-1\right)$, where $w_{j}(k)$ is the number of stops of vehicle $j$ at instant $k$. The MO problems in this step are the most time consuming, but they can be solved simultaneously and in parallel because they are not related with each other.

Step 2.2. Then for a given scenario $h \in \bar{h}$, considering the constraint that just one vehicle can serve each request, we obtain the Pareto set of the following MO problem:

$$
\min _{\left\{S(k), S^{1}, \ldots, S^{h_{\max }}\right\}}\left\{\sum_{j \in \bar{F}} \sum_{\ell=1}^{N} \Delta J_{j, h}^{U}(k+\ell), \sum_{j \in \bar{F}} \sum_{\ell=1}^{N} \Delta J_{j, h}^{O}(k+\ell)\right\}
$$

The solution of this MO problem is obtained with the Pareto sets from Step 2.1 by combining the $|\bar{F}|^{N}$ possible cases in a way that the current request and each future request are served by just one vehicle.

Step 2.3. Then, using the Pareto set of all the scenarios $h \in \bar{h}$, we solve the following MO problem:

$$
\min _{S_{k}^{k+N}}\left\{\sum_{j \in \bar{F}} \sum_{h \in \bar{h}} \sum_{\ell=1}^{N} p_{h} \cdot \Delta J_{j, h}^{U}(k+\ell), \sum_{j \in \bar{F}} \sum_{h \in \bar{h}} \sum_{\ell=1}^{N} p_{h} \cdot \Delta J_{j, h}^{O}(k+\ell)\right\}
$$

The solution of this MO problem is obtained using the Pareto sets from Step 2.2, by multiplying each Pareto front by the probability of occurrence of the associated scenario $p_{h}$ and then combining the different cases considering all the scenarios.
Step 2.4. The Pareto front is presented to the operator, who will select a sequence $S(k)$ that is Pareto optimal, based on a criterion. For example, the operator can choose the solution that provides the minimum user effective cost, or other characteristics that could be estimated. For estimating for example the effective user waiting time, we weight the expected waiting time of each of scenario with its probability of occurrence $p_{h}$. In this step the performance of the DPDP will depend on how good the criterion applied is.

In this kind of problems, HMO-MPC suits very well, as its main objective is to be implemented as a reference to support the decisions of the dispatcher, who has the flexibility of deciding which criterion is more adequate.

\section{SIMULATION RESULTS}

A period of four hours representative of a labor day (14:00-17:59) is simulated, over an urban area of approximately $81\left(\mathrm{~km}^{2}\right)$. A fixed fleet of twenty vehicles is considered, with a capacity of four passengers each. We assume that the vehicles travel in a straight line between stops and that the transport network behaves according to a speed distribution with mean equal to $20(\mathrm{~km} / \mathrm{h})$. We suppose that the future calls are unknown for the dispatcher. However, he(she) has historical data from where the typical trip patterns can be extracted. A speed distribution model and the trip patterns are known, from the historical data and the fuzzy zoning method. This fuzzy zoning permits to generate the trip patterns and their probabilities as shown in Fig. 3.

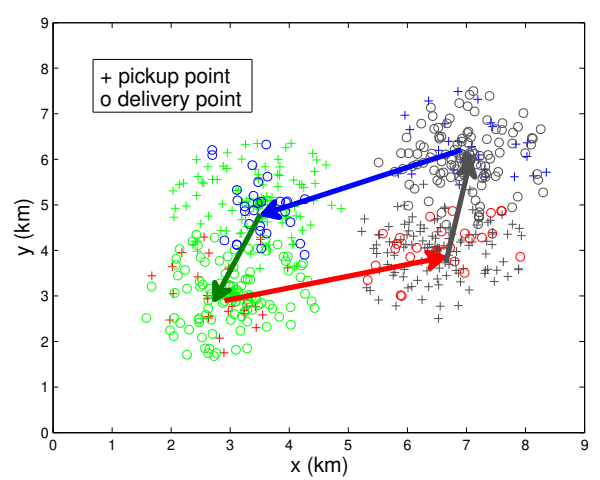

Fig. 3. HMO-MPC for the DPDP. Origin-destination patterns

Three hundred calls were generated over the simulation period of four hours following the spatial and temporal distribution observed from the historical data. A negative exponential distribution for time intervals between calls with rate $0.8(\mathrm{call} / \mathrm{min})$ was assumed. The pickup and delivery coordinates were generated randomly within each zone. The first 30 calls at the beginning and the last 30 calls at the end of the experiments were deleted from the statistics to avoid limit distortion (warm up period). One experiment was carried out to obtain the statistics, to show how the approach works. The experiment (emulating four hours and 300 online decisions) took $7.66(\mathrm{~min})$, on average 1.69 seconds per request, on a Intel Core $2 \mathrm{CPU}, 3.00 \mathrm{GHz}$ processor. 
TABLE I

SIMULATION RESULTS, USER AND OPERATOR COST

\begin{tabular}{ccccc}
\hline Case & $\begin{array}{c}\text { Effective } \\
\text { travel time } \\
(\mathrm{min})\end{array}$ & $\begin{array}{c}\text { Effective } \\
\text { waiting time } \\
(\mathrm{min})\end{array}$ & $\begin{array}{c}\text { User } \\
\text { cost } \\
(\mathrm{Ch} . \$)\end{array}$ & $\begin{array}{c}\text { Operator } \\
\text { cost } \\
(\mathrm{Ch} . \$)\end{array}$ \\
\hline a) & 11.18 & 5.82 & 477.49 & 18124.56 \\
\hline b) & 12.88 & 6.51 & 539.91 & 17499.78 \\
\hline c) & 12.68 & 8.57 & 639.62 & 16910.75 \\
\hline d) & 12.70 & 11.39 & 781.07 & 16670.66 \\
\hline
\end{tabular}

TABLE II

USER INDEXES

\begin{tabular}{cccc}
\hline Case & $\begin{array}{c}\text { Waiting time } \\
\text { higher than } \\
10 \min (\text { pax })\end{array}$ & $\begin{array}{c}\text { Unfavorable } \\
\text { total time } \\
(\text { pax })\end{array}$ & $\begin{array}{c}\text { Worst } \\
\text { served } \\
\text { user cost }(\text { Ch.\$) }\end{array}$ \\
\hline a) & 31 & 20 & 1679.82 \\
\hline b) & 50 & 32 & 2000.56 \\
\hline c) & 73 & 51 & 2574.48 \\
\hline d) & 120 & 72 & 3256.77 \\
\hline
\end{tabular}

This computing time represents an upper bound of what is possible to do if a more efficient algorithm like the metaheuristics from the multiobjective evolutionary computation were applied.

The objective function is formulated at two steps ahead, considering parameters $\theta_{v}=16.7(\mathrm{Ch} . \$ / \mathrm{min}), \theta_{e}=$ $50(\mathrm{Ch} . \$ / \mathrm{min}), c_{T}=25(\mathrm{Ch} . \$ / \mathrm{min}), c_{L}=350(\mathrm{Ch} \$ \mathrm{~km})$. The users will start to get annoyed if their perceived total travel time is bigger than 1.7 times their minimal travel time, or if their waiting time is longer than $10(\mathrm{~min})$. In the $3 \mathrm{rd}$ layer, the six best vehicles to serve a new request are chosen, ranked according the fuzzy inference system. The most likely demand pattern was used for the predictions. The formulation considers concepts already presented in the literature, like the total service time and dissatisfaction [4], the operational cost like in [8]; being the aim of this paper to present a general framework, where different objective functions could be included.

The criteria for selecting a Pareto solution was the value nearest to a given user cost. We considered four cases: $500,600,700$ and $800($ Ch.\$) for cases a), b), c) and d) respectively. Simulations for two steps ahead were conducted to analyze and evaluate the performance of the HMO-MPC strategies. In Table I the effective user waiting and travel time, user and operator costs are reported. In Table II we also show the number of passengers (pax) badly served, i.e., having a waiting time higher than a threshold of $10(\mathrm{~min})$, as well as a very bad level of service considering the total time spent in the trip (in-vehicle and waiting time) and the worst served user cost. Tables I and II clearly show the trade off between opposite components. The resulting mean user cost over the whole simulation fitted quite well the thresholds defined at each case.

\section{CONCLUSIONS AND FUTURE WORK}

A new approach to solve a dynamic pickup and delivery problem was presented. The proposed hierarchical multiobjective model predictive control scheme considers three layers. In the first one, variables with a long-term effect in the system are determined. In the second layer, the vehicles are characterized by fuzzy membership functions, which are used in the next layer to optimize the composition of the fleet in a better way. The last layer consists of an MOMPC problem. Under the implemented on-line system it is easier and more transparent for the operator to follow service policies as weighting parameters are not tuned.

The method we use in this paper has one main drawback: obtaining the solution set of the MO problem requires a significant computational effort. We claim that new toolboxes for Evolutionary Computation and other efficient algorithms (see e.g. [15]) that have been developed in recent years, make it possible to determine a good representative pseudo-optimal Pareto set in a dynamic context. Future work will thus focus on efficient optimization algorithms.

\section{ACKNOWLEDGMENT}

Research supported by the European 7th framework STREP project "Hierarchical and distributed model predictive control (HD-MPC)", Fondecyt Chile Grant 1100239 and the Millennium Institute Complex Engineering Systems (ICM:P-05-004-F, CONICYT:FBO16).

\section{REFERENCES}

[1] J. Desrosiers, F. Soumis and Y. Dumas, "A dynamic programming solution of a large-scale single-vehicle dial-a-ride with time windows", American Journal of Mathematical and Management Sciences, vol. 6, 1986, pp 301-325.

[2] M. Savelsbergh and M. Sol, "The general pickup and delivery problem", Transportation Science, vol. 29, 1995, pp 17-29.

[3] G. Berbeglia, J. Cordeau and G. Laporte, "Dynamic pickup and delivery problems", European Journal of Operational Research, vol. 202, 2009, pp 8-15.

[4] H. Psaraftis, "A dynamic programming solution to the single many-tomany immediate request dial-a-ride problem”, Transportation Science, vol. 14, 1980, pp 130-154.

[5] J. Cordeau and G. Laporte, "The dial-a-ride problem: Models and algorithms", Annals of Operations Research, vol. 153, 2007, pp 2946.

[6] R. Dial, "Autonomous dial-a-ride transit: Introductory overview", $\mathrm{Tr}$. Research Part C: Emerging Technologies, vol. 3, 1995, pp 261-275.

[7] S. Ichoua, M. Gendreau and J.Y. Potvin, "Exploiting knowledge about future demands for real-time vehicle dispatching", Transportation Science, vol. 40, 2006, pp 211-225.

[8] C.E. Cortés, D. Sáez and A. Núñez, "Hybrid adaptive predictive control for a dynamic pickup and delivery problem including traffic congestion", International Journal of Adaptive Control and Signal, vol. 22, 2008, pp 103-123.

[9] R. Scattolini, "Architectures for distributed and hierarchical model predictive control - A review", Journal of Process Control, vol. 19, 2009, pp 723-731.

[10] J. Paquette, J. Cordeau and G. Laporte, "Quality of service in dial-aride operations", Computers \& Industrial Engineering, vol. 56, 2009, pp 1721-1734.

[11] K.C. Tan, C.Y. Cheong and C.K. Goh, "Solving multiobjective vehicle routing problem with demand via evolutionary computation", European Journal of Operational Research, vol. 177, 2007, pp 813839.

[12] N. Jozefowiez, F. Semet and E. Talbi, "Multiobjective vehicle routing problems", European Journal of Operational Research, vol. 189, 2008, pp 293-309. 
[13] A. Bemporad and D. Muñoz de la Peña, "Multiobjective model predictive control", Automatica, vol. 45, 2009, pp 2823-2830.

[14] E.H. Mamdani, "Applications of fuzzy logic to approximate reasoning using linguistic synthesis", IEEE Transactions on Computers, Vol. 26, No. 12, pp. 1182-1191, 1977.

[15] R. Caballero, T. Gomez, M. Luque, F. Miguel and F. Ruiz, "Hierarchical generation of Pareto optimal solutions in large-scale multiobjective systems", Computers \& Operations Research, vol. 29, 2002, pp 15371558. 